\title{
A COMPARISON OF EPIDURAL ANESTHESIA AND LUMBAR PLEXUS-SCIATIC NERVE BLOCKS FOR KNEE SURGERY
}

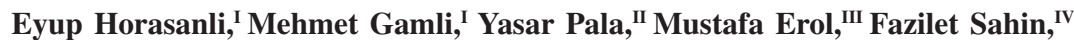 \\ Bayazit Dikmen ${ }^{1}$
}

doi: 10.1590/S1807-59322010000100006

Horasanli E, Gamli M, Pala Y, Erol M, Sahin F, Dikmen B. A comparison of epidural anesthesia and lumbar plexus-sciatic nerve blocks for knee surgery. Clinics. 2010;65(1):29-34.

OBJECTIVES: The efficacy of combined lumbar plexus-sciatic nerve blocks was compared to epidural anesthesia in patients undergoing total knee surgery.

PATIENTS AND METHODS: The study included 80 American Society of Anesthesiologists (ASA) Physical Status I-III patients (age range 18 to 65) undergoing knee surgery. The patients were randomly divided into one of two groups. Epidural anesthesia was performed in the epidural anesthesia (EA) group $(n=40)$, and the lumbar plexus and sciatic nerves were blockedin the lumbar plexus-sciatic nerve blocks (LPSB) group $(n=40)$. For each patient, onset of sensory and motor block, degree of motor block, sign of sensory block in the contralateral lower limb for the lumbar plexus-sciatic nerve blocks group, success in providing adequate anesthesia, hemodynamic changes, time of first analgesic request, and patient and surgeon satisfaction with the anesthetic technique were recorded.

RESULTS: One patient in the epidural anesthesia group and three patients in the lumbar plexus-sciatic nerve blocks group required general anesthesia due to failed block. There were no significant differences between the two groups regarding the success of providing adequate anesthesia. Eight patients in the lumbar plexus-sciatic nerve blocks group developed contralateral spread. The onset of sensory-motor block and the time of the first analgesic request were significantly later in the lumbar plexus-sciatic nerve blocks group than in the epidural anesthesia group. Although there were no significant differences regarding patient satisfaction with the anesthetic technique between the two groups, surgeon satisfaction was significantly higher in the lumbar plexus-sciatic nerve blocks group than in the epidural anesthesia group.

CONCLUSION: The lumbar plexus -sciatic nerve blocks provide effective unilateral anesthesia and may offer a beneficial alternative to epidural anesthesia in patients undergoing total knee surgery.

KEYWORDS: Epidural anesthesia; lumbar plexus; sciatic nerve block; knee surgery.

\section{INTRODUCTION}

For patients undergoing elective knee surgery, postoperative anesthesia-related pulmonary complications

\footnotetext{
${ }^{\text {I }}$ Department of Anesthesiology and Reanimation, Ankara Numune Training and Research Hospital - Ankara, Turkey.

II Anesthesiology and Reanimation, Cerkezkoy Optimed Guven Hospital Tekirdag, Turkey.

III Anesthesiology and Reanimation, Bingol State Hospital - Bingol, Turkey.

${ }^{\text {Iv }}$ Anesthesiology and Reanimation, Nigde State Hospital - Nigde, Turkey.

Email: eyuphorasanli@yahoo.com

Tel: +90312 5084229 - +90506 6002216

Received for publication on August 17, 2009

Accepted for publication on October 15, 2009
}

and confusion can interfere with recovery, timely discharge, and participation in early physical therapy. For these reasons, the use of regional anesthesia, including either central blocks or multiple peripheral nerve blocks, has increased. ${ }^{1-4}$ These blocks also ensure adequate intraoperative and postoperative analgesia and anesthesia. ${ }^{2}$

Recently, among these regional anesthesia techniques on the lower limb, peripheral nerve blocks are gaining popularity because they reduce the possibility of complications and side effects associated with the central blocks. Peripheral nerve blockade provides effective analgesia and anesthesia with potentially fewer 
complications and side effects than central blocks. ${ }^{2,5-7}$

The three main nerves of the lumbar plexus contribute to the innervation of the lower limb. Blockade of these nerves, combined with the sciatic blockade, can produce complete blockade of the lower limb. ${ }^{5}$

Ropivacaine is a well-tolerated regional anesthetic effective for surgical anesthesia as well as the relief of postoperative and labor pain. Thus, ropivacaine, with its efficacy, lower propensity for motor block, and reduced potential for central nervous system toxicity and cardiotoxicity, appears to be a better option for regional anesthesia. ${ }^{6,7}$

The hypothesis of the present study is that lumbar plexus - sciatic nerve blocks, achieve adequate analgesia and anesthesia as compared to epidural anesthesia in patients undergoing knee surgery.

\section{MATERIALS AND METHODS}

After approval by the hospital's Ethics Committee and written informed patient consent, 80 American Society of Anesthesiologists (ASA) Physical Status I-III patients (age range 18 to 65 ) undergoing elective unilateral knee surgery were randomly allocated into one of two groups using sealed envelopes. The epidural anesthesia was performed in Group Epidural Anesthesia (EA) ( $n=40)$, and the lumbar plexus and sciatic blocks were performed in Group Lumbar Plexus-Sciatic Nerve Blocks (LPSB) $(n=40)$. The same anesthesiologist, who was experienced in both block techniques, performed all procedures in both groups. Tourniquets were used in all patients in both the EA and LPSB groups. Exclusion criteria for both groups included contraindications to epidural anesthesia, peripheral blocks, or anesthetics used; evidence of severe cardiovascular, renal, hematological or hepatic diseases; preexisting neurological or psychiatric illnesses; chronic pain syndromes; alcohol or drug abuse; and mental retardation.

Before all procedures, an 18-gauge cannula was inserted into the basilic vein, followed by routine, continuous monitoring that consisted of electrocardiography (ECG), heart rate, and peripheral oxygen saturation $\left(\mathrm{SpO}_{2}\right)$ monitoring. Noninvasive arteriel blood pressure was measured at 5-min intervals (Datex- Ohmeda ADU ${ }^{\circledR}$, GE Healthcare, Madison, USA).For each patient , $10 \mathrm{ml} / \mathrm{kg}$ of intravenous (IV) isotonic saline were administered as a preload $30 \mathrm{~min}$ before regional block procedures.

Lumbar plexus blockade was performed using a posterior paravertebral approach at the L3 vertebral level. ${ }^{8}$ Patients were laid in the lateral decubitus position (Sim's position), with the operative side facing up. The needle insertion point was located four centimeters lateral to the spinous process of L3 . Under full aseptic conditions, $3 \mathrm{ml}$ of $2 \%$ lidocaine were injected into this point subcutaneously. A Stimuplex $A^{\circledR}$ 150-mm needle (Braun Medical, Melsungen, Germany) was inserted and connected to the nerve stimulator (Stimuplex $\mathrm{S}^{\circledR}$, B.Braun Medical, Germany) with a starting output of $1.5 \mathrm{~mA}$ and $2 \mathrm{~Hz}$.

The needle was inserted four centimeters lateral to the spinous process of L3 and directed slightly cephalic (but not medially or laterally) to contact the transverse process of L3. After touching the process, the needle was than redirected caudally to the transverse process and advanced $1.5 \mathrm{~cm}$ deeper. Contraction of the quadriceps muscle in response to electrical stimulation was used to identify the proximity to the plexus, and the needle was advanced until muscle twitches were elicited with currents between 0.3 and $0.5 \mathrm{~mA}$ at $2 \mathrm{~Hz}$. After negative aspiration, $30 \mathrm{ml}$ of $0.375 \%$ ropivacaine were injected

The sciatic nerve block was based on Labat's technique. ${ }^{5}$ In the Sim's position, the same needle was inserted at a right angle to all cutaneous planes at the caudal end of a $3-5-\mathrm{cm}$ line originating from, and perpendicular to, the middle of a line that intersects the great trochanter posterior to the iliac spine. In each case, neural structures were identified with the help of a nerve stimulator using a stimulus of $1.5 \mathrm{~mA}$ at $2 \mathrm{~Hz}$, while contractions of the gastrocnemius (foot plantar flexion) and/or tibialis anterior (foot dorsi-flexion) indicated proximity to the sciatic nerve and the needle was introduced until muscle twitches were elicited with currents between 0.3 and $0.5 \mathrm{~mA}$ at $2 \mathrm{~Hz}$. After negative aspiration, $20 \mathrm{ml}$ of $0.375 \%$ ropivacaine were injected. Patients were then returned to a semirecumbent position with a head-up angle of $30-45^{\circ}$ with respect to the operating table. ${ }^{9}$

For each block, onset of nerve blockade was evaluated every $2.5 \mathrm{~min}$ and evaluations continued for 40 minutes after completion of the nerve blocks. If no sensory and motor block were present after this time, patients were excluded from the study, and their surgery proceeded with general anesthesia. In addition, signs of sensory block were also sought for in the other lower extremity. Sensory evaluation using a blunt 21-gauge needle consisted of loss of pinprick sensation in the sciatic (sole of foot), femoral (anterior thigh), lateral cutaneous (lateral thigh), and obturator (medial thigh) nerve territories. The motor block was evaluated by testing knee extension (femoral nerve), thigh adduction (obturator nerve), dorsi-flexion, and plantar flexion of the foot (common peroneal and tibial nerves). The motor block was also assessed by a modified Bromage Score ${ }^{5}$ Scale (0-3): 0 , no motor impairment (able to move hip, knee, and ankle joints); 1, unable to raise either extended leg (able to move joints of knee and ankle); 2, unable to raise extended leg and flex knee (able to move joint of ankle); 3, unable to move 
knee and foot. The onset of sensory and motor block was defined as the time from completion of the lumbar plexus and sciatic nerve block to the occurrence of sensory and motor block at the related nerve territories.

Epidural anesthesia was performed in a standard fashion at the L3-L4 interspace with the operative knee in the dependent position. Under full aseptic conditions, skin infiltration was performed with $2 \mathrm{ml}$ of $2 \%$ lidocaine. An 18-G Tuohy needle with a $20-\mathrm{G}$ catheter (Perifix, B.Braun, Germany) was inserted through the L3-4 interspace, and the epidural space was located using the loss of resistance technique. The catheter was then advanced approximately 3 $\mathrm{cm}$ cephalic. A test dose of $3 \mathrm{ml}$ of $2 \%$ lidocaine containing epinephrine (freshly added) in a ratio of 1:200,000 was administered to detect intrathecal or IV injection. After negative response, $15 \mathrm{ml}$ of $0.75 \%$ epidural ropivacaine which is $112.5 \mathrm{mg}$ were administered, and the patient was turned to the supine position. The speed of epidural ropivacaine administration was $3 \mathrm{ml} / 10 \mathrm{~s}$. In addition, the sensory level of the epidural block was assessed by the loss of pinprick method at 2.5 -min intervals for $40 \mathrm{~min}$ after completion of the block. Pinprick sensation was examined using a blunt 21-gauge needle in a cephalicto-caudal fashion along the left anterior axillary line. The onset of sensory block was defined as the time from epidural injection to the occurrence of sensory block at the T10 dermatome. The motor block was assessed at 2.5-min intervals for $40 \mathrm{~min}$ after completion of epidural block using a modified Bromage Score Scale. The onset of motor block was defined as the time from epidural injection to the occurrence of motor block at each scale. The duration of sensory blocks (the time interval between the procedure and the need for the first analgesic requirement) was noted.

Arterial blood pressure and heart rate were recorded every 5 min after all procedures. Hypotension (systolic blood pressure $<100 \mathrm{~mm} \mathrm{Hg}$ or a decrease of more than $30 \%$ from baseline) was treated with $10 \mathrm{mg}$ of IV ephedrine as needed. Side effects such as nausea, vomiting, hypotension, or bradycardia were recorded during surgery and $24 \mathrm{~h}$ postoperatively. Further sedation was provided during surgery with an IV bolus of midazolam $(0.03-0.05 \mathrm{mg} / \mathrm{kg})$ and a continuous infusion of propofol ( 1 to $2 \mathrm{mg} / \mathrm{kg} / \mathrm{hr}$ ) for the patients who were experiencing discomfort and/or were anxious at any time during the surgical procedure.

Patient and surgeon satisfaction with the anesthetic techniques was evaluated at the end of surgery using a two-point score: 1, satisfactory (if necessary, I would have the same anesthetic again); and 2, unsatisfactory (different anesthetic).

For each patient, the following data were collected: weight, age, sex, site of surgery, onset of sensory and motor block, degree of motor block, sign of sensory block in the contralateral lower limb for the LPSB group, success in providing adequate anesthesia, hemodynamic changes, time to first analgesic requirement (FAT), and patient and surgeon satisfaction.

Statistical analysis: All data were checked for a normal distribution using the Shapiro-Wilk test. Quantitative variables were compared between groups using the Student's t-test or Mann-Whitney U-test, depending on whether normal or non-normally distributed variables were used, respectively. Differences with respect to demographic data (age, weight, sex distribution) between the two groups were analyzed using the Student's t-test. Onset of sensory and motor block, first analgesic request time, and motor block regression time were analyzed by the Mann-Whitney U-test. Differences in duration of postoperative analgesia, incidences of complications, and patient and surgeon satisfaction were analyzed with the chisquare test. Data were analyzed using SPSS 11.5 software (SPSS, Chicago, IL, USA). A P value less than 0.05 was considered statistically significant.

\section{RESULTS}

Eighty patients were included in this study, equally divided over the two intervention groups. One patient in the EA group and three patients in the LPSB group required general anesthesia due to failure of the regional technique. These four patients were excluded from the study. Thus, the success of providing adequate anesthesia was $97.5 \%$ (39 patients) in the EA group and $92.5 \%$ (37 patients) in the LPSB group $(\mathrm{p}>0.05)$. There were no significant differences between the two groups in terms of demographic data (age, sex, weight, and height), ASA physical status, and duration of surgery (Table I).

Eight patients (21.6\%) in the LPSB group developed contralateral spread. The onset of sensory block was

Table 1 - Demographic data, ASA classification, and duration of surgery for the two groups of patients expressed as mean $\pm \mathrm{SD}$ or number of patients per category

\begin{tabular}{lccc}
\hline & $\begin{array}{c}\text { Group EA } \\
\mathrm{n}=39\end{array}$ & $\begin{array}{c}\text { Group LPSB } \\
\mathrm{n}=37\end{array}$ & P-value \\
\hline Age (years) & $54.0 \pm 16.9$ & $51.3 \pm 14.9$ & $\mathrm{P}>0.05$ \\
Sex (F/M) & $12 / 27$ & $13 / 24$ & $\mathrm{P}>0.05$ \\
Weight $(\mathrm{kg})$ & $77.8 \pm 12.3$ & $78.5 \pm 10.5$ & $\mathrm{P}>0.05$ \\
Height $(\mathrm{cm})$ & $163.6 \pm 5.1$ & $165.1 \pm 10.0$ & $\mathrm{P}>0.05$ \\
ASA I / II/ III & $14 / 29$ & $14 / 23$ & $\mathrm{P}>0.05$ \\
$\begin{array}{l}\text { Duration of sur- } \\
\text { gery (min) }\end{array}$ & $85.9 \pm 43.8$ & $81.1 \pm 34.1$ & $\mathrm{P}>0.05$ \\
\hline
\end{tabular}

ASA, American Society of Anesthesiologists physical status; EA, epidural anesthesia; LPSB, lumbar plexus combined sciatic block 
significantly later in the LPSB group (median: $13 \mathrm{~min}$ ) compared to the EA group (median: $10 \mathrm{~min})(\mathrm{p}<0.05)$.

The onset of motor block was significantly later in the LPSB group (median: $18 \mathrm{~min}$ ) than in the EA group (median: $14 \mathrm{~min})(\mathrm{p}<0.05)$. There were no differences between the two groups regarding degree of motor block $(\mathrm{p}>0.05)$. The time from block placement until first request for analgesia (the duration of analgesia) varied between 150 and 560 min (median: $240 \mathrm{~min}$ ) for the EA group, while it took between 240 and $720 \mathrm{~min}$ (median: $360 \mathrm{~min}$ ) for the LPSB group.

Consequently, the time to first analgesic requirement was significantly later in the LPSB group than in the EA group $(p<0.05)$. The time of motor block regression (modified Bromage score 3 to 1 ) occurred earlier in group EA (median: $105 \mathrm{~min}$ ) as compared to group LPSB (median: $140 \mathrm{~min}$ ) (p $<0.05$ ).

We also found that EA with $0.75 \%$ ropivacaine (30 patients) produced less motor blockade (modified Bromage score $\leq 2)$ than LPSB with $0.375 \%$ ropivacaine (24 patients) during the intraoperative period $(\mathrm{p}<0.05)$. These results are shown in Tables II and III.

Eleven patients in the EA group showed an intraoperative modified Bromage score $<2$ as compared with only eight patients in the LPSB group. Complete motor block (modified Bromage score $\geq 2$ ) was observed in 28 patients in the EA group and 29 patients in the LPSB group. In addition, six patients from the EA group and four patients from the LPSB group required intraoperative sedation.

The number of patients who required additional sedation was higher in the EA group (eleven patients) compared

Table 2 - Characteristics of regional anesthetic techniques expressed as median (min-max) or number of patients per category

\begin{tabular}{lccc}
\hline & $\begin{array}{c}\text { Group EA } \\
\mathrm{n}=39\end{array}$ & $\begin{array}{c}\text { Group LPSB } \\
\mathrm{n}=37\end{array}$ & P-value \\
\hline Onset of sensory block (min) & 10 & 13 & $\mathrm{p}<0.05$ \\
& $(5-25)$ & $(5-27.5)$ & \\
Onset of motor block (min) & 14 & 18 & $\mathrm{p}<0.05$ \\
& $(7.5-27.5)$ & $(10-25)$ & \\
Contralateral extension & - & 8 & \\
First analgesic request time (min) & 240 & 360 & \\
& $(150-560)$ & $(240-720)$ & $\mathrm{p}<0.05$ \\
Motor block regression (min) & 105 & 140 & $\mathrm{p}<0.05$ \\
$\begin{array}{l}\text { Satisfaction with anesthetic technique: } \\
\text { Patients: satisfactory / }\end{array}$ & $29 / 125)$ & $(120-260)$ & \\
unsatisfactory & & $28 / 8$ & $\mathrm{p}>0.05$ \\
$\begin{array}{l}\text { Surgeons: satisfactory / } \\
\text { unsatisfactory }\end{array}$ & $26 / 13$ & $30 / 7$ & $\mathrm{p}<0.05$ \\
\hline
\end{tabular}

EA, epidural anesthesia; LPSB, lumbar plexus combined with sciatic block.
Table 3 - Degree of motor block (modified Bromage Score Scale) expressed as number of patients and percentage per category

\begin{tabular}{lccc}
\hline & $\begin{array}{c}\text { Group EA } \\
\mathrm{n}=39\end{array}$ & $\begin{array}{c}\text { Group LPSB } \\
\mathrm{n}=37\end{array}$ & P-value \\
\hline 1, unable to raise extended leg & $11(28.20 \%)$ & $8(21.62 \%)$ & $\mathrm{P}>0.05$ \\
2, unable to raise extended leg & $19(48.72 \%)$ & $16(43.24 \%)$ & $\mathrm{P}>0.05$ \\
$\begin{array}{l}\text { and flex knee } \\
\text { 3, unable to move knee and foot }\end{array}$ & $9(23.08 \%)$ & $13(35.14 \%)$ & $\mathrm{P}<0.05$ \\
\hline
\end{tabular}

EA, epidural anesthesia; LPSB, lumbar plexus combined with sciatic block.

with the group LPSB (eight patients). However, there were no significant differences between the number of patients requiring sedation( $p>0.05)$. In 11 patients, analgesia was adequate until a specified time. In most of these patients, discomfort occurred when the surgeon started hammering for the purpose of fitting the prosthesis to the bones. In some patients, this was also the moment when satisfactory neuromuscular block changed to unsatisfactory. Intravenous midazolam and propofol were effective in providing sedation in these patients. Mean propofol consumption was $54.5 \pm 4.7$ $\mathrm{mg}$ in the EA group and $56.3 \pm 3.5 \mathrm{mg}$ in the LPSB group $(\mathrm{p}=0.648)$. Mean midazolam consumption was $3.6 \pm 0.3$ $\mathrm{mg}$ in the EA group and $3.5 \pm 0.2 \mathrm{mg}$ in the LPSB group $(\mathrm{p}=0.210)$. The use of midazolam and propofol was similar in both groups $(\mathrm{p}>0.05)$.

Changes in arterial blood pressure and heart rate were not different between the two groups ( $p>0.05$ ).

The overall level of patient satisfaction with the anesthetic procedure was lower in the LPSB group than in the EA group. Twenty-nine patients (78.4\%) in the EA group and twenty-eight patients (75.7\%) in the LPSB group were satisfied with the anesthetic technique. However, there were no significant differences between the two groups $(\mathrm{p}>0.05$ ) (Table III). Surgeon satisfaction was significantly higher in the LPSB group (81\%) than in the EA group (66.6\%) $(\mathrm{p}<0.05)$. In eleven cases in the EA group and eight cases in the LPSB group, surgeons complained of inadequate motor blockade (modified Bromage score $<2$ ). Therefore, these patients required propofol sedation (Table III).

No severe events were reported in any of the patient. There was no difference in frequency of complications between the two groups $(p>0.05)$. Vasovagal reflex occurred in one patient in the EA group. Nevertheless, hypotension (nine patients in the EA group and five patients in the LPSB group) and bradycardia (four patients in the EA group and two patients in the LPSB group) occurred in each group. The decrease in arterial blood pressure necessitated $10 \mathrm{mg}$ of IV ephedrine (nine patients in the EA group and five patients in the LPSB group). 
Bradycardia was treated with $0.5 \mathrm{mg}$ of IV atropine. One patient in the EA group experienced vomiting; three patients in the EA group and one patient in the LPSB group experienced nausea. No patient demonstrated any clinical symptoms or signs of local anesthetic toxicity (Table IV).

Table 4 -The frequency of complications after completion of regional anesthetic techniques expressed as number of patients and percentage per category

\begin{tabular}{lcc}
\hline & Group EA n=39 & Group LPSB $\mathrm{n}=37$ \\
\hline Vasovagal reflex & $1(2.56 \%)$ & 0 \\
Hypotension & $9(23.08 \%)$ & $5(13.51 \%)$ \\
Bradycardia & $4(10.26 \%)$ & $2(5.41 \%)$ \\
Nausea & $3(7.69 \%)$ & $1(2.70 \%)$ \\
Vomiting & $1(2.56 \%)$ & 0 \\
Toxicity of local anesthetic & 0 & 0 \\
\hline
\end{tabular}

EA, epidural anesthesia; LPSB, lumbar plexus combined with sciatic block.

\section{DISCUSSION}

This study showed that performing LPSB with $0.375 \%$ ropivacaine provides effective anesthesia with few complications in comparison with epidural anesthesia. The duration of analgesia in the LPSB group was longer than in the EA group; however, the onset of sensory and motor block was significantly later in the LPSB than in the EA group.

In particular, L2-3 approaches were potentially preferable to L4-5 approaches because the L2-3 interspace is wider, reducing the likelihood of complications. Despite the small distances between the medial and lateral borders of the psoas major at this level, neurostimulation can be used to indicate plexus proximity. ${ }^{10}$ Consequently, lumbar plexus blockade was performed using a posterior paravertebral approach at L3 in our study.

Chayen et al. ${ }^{11}$ demonstrated that the success in providing adequate anesthesia using a lumbar plexus block (L4-5 approach) for orthopedic surgery of the lower extremity was $90 \%$ patient. This result is consistent with the experience of Farny et al. ${ }^{12}$ (89\% patient, 40/45). Parkinson and colleagues performed lumbar plexus block via Dekrey's L3 approach (25 patients) and L4-5 approach (23 patients) and reported anesthetic conditions for surgery were achieved in 24 patients (96\%) using the L3 approach and 21 patients (91\%) using the L4-5 approach. ${ }^{8}$ In this study, we demonstrated that the success in providing adequate anesthesia was $92.5 \%$ in the LPSB group. Our results were similar to these findings.

Contralateral extension of the analgesia suggesting an epidural distribution of the local anesthetic is a well- recognized complication of the posterior technique of the lumbar plexus block. The incidence of bilateral block varies according to the technique used. Parkinson et al. found contralateral spread in $16 \%$ of patients using motor testing after a psoas compartment block performed via Dekrey's L3 approach. ${ }^{10}$ However, Biboulet et al., performing Dekrey's L3 approach technique, detected contralateral spread in $26.6 \%$ of patients using sensory evaluation. ${ }^{11,15} \mathrm{We}$ demonstrated that the extent of the success of lumbar plexus blockade using the posterior approach at L3 was $21.6 \%$ (8 of the 40 patients) with sensory evaluation in this study. Our study demonstrated similar results with the recent literature.

The onset of sensory and motor block for ropivacaine at LPSB in our study was consistent with the experiences of Greengrass et al. and Piangatelli et al. ${ }^{6,7}$ The onset of sensory and motor block during epidural ropivacaine anesthesia in our study was similar to the other studies..$^{14,15}$ Nevertheless, the onset of sensory block for EA was relatively later in the study of Casati et al. as compared to our study. This difference may be due to the lower concentration and volume of ropivacaine $\left(0.5 \%, 10 \mathrm{ml}\right.$, respectively) used in this study. ${ }^{16}$

The onset of sensory and motor block in the LPSB group was significantly later than in the EA group in our study. This result can be explained by the fact that local anesthetic solution must cross different anatomical barriers such as fibrous tissue and nerve sheaths before reaching the site of action in peripheral nerves. In the case of epidural block, rapid diffusion of local anesthetic towards the thin dural cuff region causes faster onset of sensory and motor block. ${ }^{17}$

The duration of analgesia in the LPSB group was relatively short in our study as compared to other studies. ${ }^{67}$ This result may be due to the lower concentration and dose of ropivacaine $(0.375 \%, 18.75 \mathrm{mg}$, respectively) in the LPSB group. In addition, Greengrass et al. performed lumbar plexus and sciatic nerve block using either ropivacaine or bupivacaine with fresh epinephrine. The addition of epinephrine causes vasoconstriction at the site of administration. The consequent decreased absorption increases neuronal uptake and prolongs duration of action. ${ }^{17}$ Therefore, the first analgesic request time also took longer in the study of Greengrass et al. ${ }^{6}$. The duration of analgesia was different in the EA group as compared to other studies. ${ }^{3,14}$ This result may be due to a different concentration or volume of ropivacaine $(0.75 \%, 15$ $\mathrm{ml}$, respectively) used for epidural anesthesia.

We found that the median time to first analgesic request for the LPSB group was significantly longer than for the EA group. The first request for analgesia is related to block regression. Regression of block occurs due to diffusion of the local anesthetic away from the site of action, which in turn depends upon the vascularity of that particular tissue. ${ }^{17}$ Rapid washout of the drug from the epidural space due to 
greater vascularity resulted in earlier block regression in the LPSB group in this study.

Turker et al. compared the epidural block and psoas compartment block for the frequency of complications after completion of these blocks. ${ }^{18}$ Hypotension, urinary retention, and nausea/vomiting were significantly more frequent in their epidural block group. Zaric et al..$^{15}$ demonstrated that the frequency of dizziness, pruritus, nausea/vomiting, and urinary retention was greater in their epidural block group ( $0.2 \%$ ropivacaine plus sufentanil) than in patients with a combined femoral and sciatic block ( $0.5 \%$ ropivacaine). Davies et al. reported that the frequency of complications (except hypotension) was similar to that the frequency of complications due to a combined femoral with sciatic block and epidural blockade. ${ }^{2}$ There was no difference in frequency of complications between the two groups in our study. Our results are consistent with those of Davies and colleagues.

Raimer et al. recorded that patient satisfaction with the anesthetic technique was high, and no significant differences were observed between psoas compartment-sciatic analgesia and epidural analgesia for postoperative pain therapy after knee arthroplasty. ${ }^{19}$ We investigated satisfaction of both the patients and the surgeons. We observed that patient satisfaction with the procedure was not significantly different between the EA and LPSB groups. However, surgeon satisfaction was significantly higher in the LPSB group.

In conclusion, the results of this prospective, randomized study demonstrated that LPSB provides effective unilateral anesthesia in patients undergoing knee surgery, with a high surgeon satisfaction rate. Hemodynamic stability, low incidence rate of complications, and patient satisfaction with the combined lumbar plexus and sciatic nerve block are similar to those variables associated with epidural anesthesia. This study suggests that the combined lumbar plexus and sciatic nerve block offers a beneficial alternative to epidural anesthesia for knee surgery.

\section{REFERENCES}

1. Liu S, Carpenter RL, Neal JM. Epidural anesthesia and analgesia: Their Role in postoperative outcome. Anesthesiology. 1995;82:1474-506.

2. Davies AF, Segar EP, Murdoch J, Wright DE, Wilson IH. Epidural infusion or combined femoral and sciatic nerve blocks as perioperative analgesia for knee arthroplasty. Br J Anaesth. 2004;93:368-74.

3. McGlade DP, Kalpokas MV, Mooney PH, Buckland MR, Vallipuram SK, Hendrata MV, et al. Comparison of $0.5 \%$ ropivacaine and $0.5 \%$ bupivacaine in lumbar epidural anaesthesia for lower limb orthopaedic surgery. Anaesth Intensive Care. 1997;25:262-6.

4. Jankowski CJ, Hebl JR, Stuart MJ, Rock MG, Pagnano MW, Beighley $\mathrm{CM}$, et al. A comparison of psoas compartment block and spinal and general anesthesia for outpatient knee arthroscopy. Anesth Analg. 2003;97:1003-9.

5. Bridenbaugh PO, Wedel DJ. The Lower Extremity: Somatic Blockade. In: Cousins MJ, Bridenbaugh PO, Editors. Neural Blockade in Clinical Anesthesia and Management of Pain. 3rd. ed. Philadelphia: LippincottRaven; 1998. p. 373-94.

6. Greengrass RA, Klein SM, D’Ercole FJ, Gleason DG, Shimer CL, Steele SM. Lumbar plexus and sciatic nerve block for knee arthroplasty: Comparison of ropivacaine and bupivacaine. Can J Anaesth. 1998;45:1094-6.

7. Piangatelli C, De Angelis C, Pecora L, Recanatini F, Testasecca D. Levobupivacaine versus ropivacaine in psoas compartment block and sciatic nerve block in orthopedic surgery of the lower extremity. Minerva Anestesiol. 2004;70:801-7.

8. Parkinson SK, Mueller JB, Little WL, Bailey SL. Extent of blockade with various approaches to the lumbar plexus. Anesth Analg. 1989;68:243-8.

9. Mannion S, O'Callaghan S, Walsh M, Murphy DB, Shorten GD. In with the new, out with the old? Comparison of two approaches for psoas compartment block. Anesth Analg. 2005;101:259-64.

10. Hanna MH, Peat SJ, D'Costa F. Lumbar plexus block: an anatomical study. Anaesthesia. 1993;48:675-8.
11. Chayen D, Nathan H, Chayen M. The Psoas Compartment Block. Anesthesiology. 1976;45:95-9.

12. Farny J, Girard M, Drolet P.Posterior approach to the lumbar plexus combined with a sciatic nerve block using lidocaine. Can J Anaesth. 1994;41:486-91.

13. Biboulet P, Morau D, Aubas P, Bringuier-Branchereau S, Capdevila $\mathrm{X}$. Postoperative analgesia after total-hip arthroplasty: Comparison of intravenous patient-controlled analgesia with morphine and single injection of femoral nerve or psoas compartment block. a prospective, randomized, double-blind study. Reg Anesth Pain Med. 2004;29:102-9.

14. Salgado PF, Sabbag AT, da Silva PC, Brienze SL, Dalto HP, Módolo NS, et al. Synergistic effect between dexmedetomidine and $0.75 \%$ ropivacaine in epidural anesthesia. Rev Assoc Med Bras. 2008;54:110-5.

15. Zaric D, Axelsson K, Nydahl PA, Philipsson L, Larsson P, Jansson JR. Sensory and motor blockade during epidural analgesia with $1 \%, 0.75 \%$, and 0.5\% ropivacaine-a double-blind study. Anesth Analg. 1991;72:509-15.

16. Casati A, Santorsola R, Aldegheri G, Ravasi F, Fanelli G, Berti M, et al. Intraoperative epidural anesthesia and postoperative analgesia with levobupivacaine for major orthopedic surgery: a double-blind, randomized comparison of racemic bupivacaine and ropivacaine. J Clin Anesth. 2003;15:126-31.

17. Tucker GT, Mather LE. Properties, Absorption, and Disposition of Local Anesthetic Agents. In: Cousins MJ, Bridenbaugh PO, Editors. Neural Blockade in Clinical Anesthesia and Management of Pain. 3rd. ed. Philadelphia: Lippincott-Raven; 1998. p. 55-95.

18. Türker G, Uçkunkaya N, Yava çao lu B, Yilmazlar A, Ozçelik S. Comparison of the catheter-technique psoas compartment block and the epidural block for analgesia in partial hip replacement surgery. Acta Anaesthesiol Scand. 2003;47:30-6.

19. Raimer C, Priem K, Wiese AA, Birnbaum J, Dirkmorfeld LM, Mossner A, et al. Continuous psoas and sciatic block after knee arthroplasty: good effects compared to epidural analgesia or i.v. opioid analgesia: a prospective study of 63 patients. Acta Orthop. 2007;78:193-200. 